\title{
Contribution des méthodes de lutte mécanique au désherbage intégré des grandes cultures oléagineuses
}

\author{
Jean LIEVEN ${ }^{1}$ \\ Jean-Louis LUCAS $^{2}$ \\ ${ }^{1}$ CETIOM, Direction technique, \\ Centre de Grignon, avenue Lucien Bretignières, \\ 78850 Thiverval-Grignon \\ $<$ lieven@cetiom.fr> \\ ${ }^{2}$ CETIOM, Bureau de la Zone Est, ZAE Capnord, \\ 10 avenue de Dallas, 21000 Dijon \\ <lucas@cetiom.fr>
}

\begin{abstract}
The integrated approach of weed management refers to a combination of direct and indirect methods in order to limit chemical applications. In oilseed rape, sunflower and soybean, mechanical weed control can be an alternative or a complement to herbicides and should take progressively a larger place in the direct weed control options, taking into account of the policy of restriction of chemical use. CETIOM launched a research program to assess, in different backgrounds, the selectivity and the efficacy of three tools, used either alone or combined with herbicides: the rotary hoe, the finger weeder and the in-row hoe. As an alternative or a complement to herbicides, mechanical weed control strategies were selective enough for oil crops if they were used in appropriate conditions (crop stage, soil surface moisture, adjustments of the tools). Linked with long term preventive measures to maintain weed population at a low level, they could be as efficient as herbicides but with an irregularity and strongly dependent on weed development stage. These techniques could be economically competitive but require often more working time. Mechanical management can thus be an answer to some of the new requirements of the agricultural context but operator skill, experience and knowledge are critical to success. Introducing these weed control methods in a farm requires a deep change of state of mind which appears today as a major restraint for the farmers.
\end{abstract}

Key words: oil crops, mechanical weed control, selectivity, efficacy

\section{Introduction}

\section{Contexte et enjeux du désherbage en grandes cultures}

Actuellement, la lutte contre les mauvaises herbes des grandes cultures repose très largement sur l'utilisation d'herbicides sélectifs. Or, depuis plusieurs années, ces pratiques de désherbage chimique sont remises en cause pour des raisons techniques, environnementales et sociétales [13]. La réduction de leur impact sur les milieux naturels constitue une des priorités mises en évidence lors du Grenelle de l'environnement, renforçant ainsi les dernières réglementations de la PAC, de la Directive-cadre sur I'Eau ou encore du Plan interministériel de réduction des risques liés aux pesticides. Les textes réglementaires incitent, entre autres, à faire adopter par les agriculteurs des pratiques plus économes en produits phytosanitaires.

La notion de « désherbage intégré » suppose que l'on passe d'une technique fondée exclusivement sur l'emploi d'herbicides à l'échelle de la culture à une lutte globale à l'échelle d'une succession de cultures [1]. Pour compenser la réduction de la pression herbicide, la modification du système doit s'accompagner de la mise en œuvre d'une combinaison de techniques à « effet partiel ». Ces dernières couvrent un large éventail allant des techniques simples, mais pas toujours réalisables - comme le décalage de la date de semis en blé - à des techniques plus complexes aux résultats aléatoires et qui font le jeu d'interactions entre le milieu, la culture et les adventices - comme l'implantation de cultures sous couverts [1-3].

\section{Le cas des oléagineux}

Les herbicides occupent une place essentielle pour l'élimination des mauvaises herbes des grandes cultures oléagineuses. Les gammes $d^{\prime}$ 'herbicides relativement étroites en colza, tournesol et soja, offrent essentiellement des matières actives à appliquer avant le semis ou la levée des cultures, ce qui impose un raisonnement systématique dans bon nombre de situations. Dans les dernières enquêtes menées par le CETIOM, pour près de $60 \%$ de la sole française de colza semée en 2007, le désherbage commence par une base de pré-semis (trifluraline ou napropamide). Ce pourcentage est similaire pour le tournesol conduit en 2006 (trifluraline, pendiméthaline, s-métolachlore). Pour le cas du soja conduit en mode conventionnel, l'enquête
2007 ramène ce chiffre à $48 \%$ (trifluraline, alachlore, s-métolachore). Pour les trois cultures, les herbicides de post-semis/prélevée, en complément ou non d'herbicide de pré-semis, demeurent prépondérants.

Sur le plan économique, les coûts des herbicides représentent environ 25 à $30 \%$ des charges opérationnelles chez le colza et chez le tournesol et entre 35 et $40 \%$ en moyenne chez le soja. Ces chiffres tendent à augmenter d'année en année, en lien notamment avec l'évolution des systèmes culturaux. En effet, le contexte socioéconomique de la dernière décennie a singulièrement favorisé les pratiques entraînant des risques de salissement des parcelles : simplification du travail du sol, abandon du labour (figure 1), renoncement au concept agronomique de la rotation. Par voie de conséquence, le désherbage uniquement chimique des oléagineux montre ses limites pour certaines espèces, appartenant notamment aux familles des graminacées, crucifères, géraniacées et astéracées. Dans le même temps, les phénomènes d'apparition et de développement des résistances aux graminicides (inhibiteurs d'ACC-ase et d'ALS) renforcent les inquiétudes dans les grands bassins de production, à l'heure où les homologations de nouvelles matières actives, a fortiori 


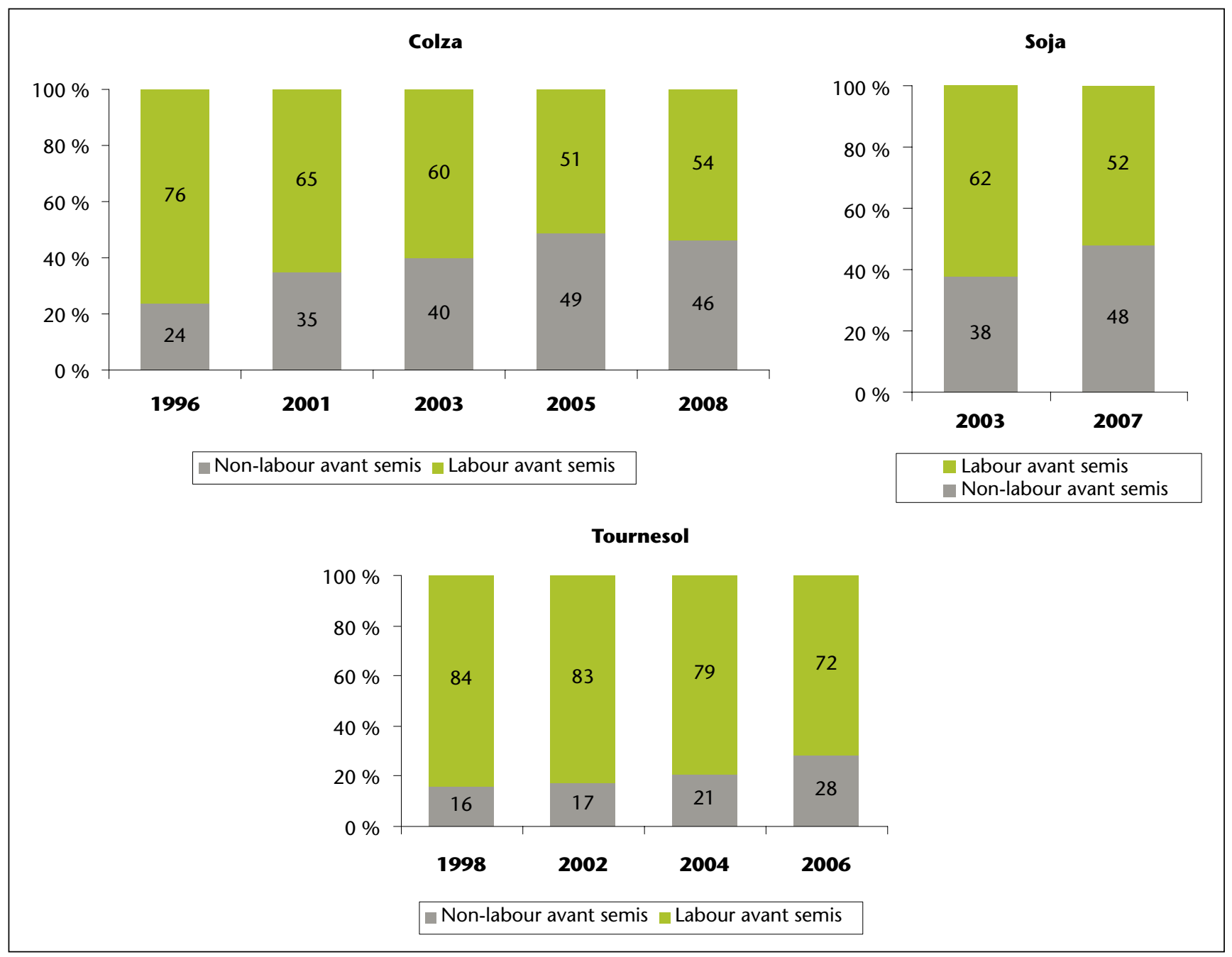

Figure 1. Évolution (en \% des surfaces) du travail du sol avant implantation de colza, tournesol et soja, enquêtes culturales CETIOM.

utilisant de nouveaux modes d'action, peinent à voir le jour.

Jusqu'en 2008, la trifluraline constituait la base du désherbage chimique des oléagineux. Son absence risque de faire émerger de nouveaux types de flores ou d'exacerber les problématiques citées ci-dessus. Enfin, il sera difficile de trouver un héritier à la trifluraline aussi bon marché et à régularité d'efficacité aussi intéressante $[4,5]$.

Pour toutes ces raisons, lorsqu'elles sont combinées à des méthodes prophylactiques adéquates, les techniques de désherbage mécanique apparaissent comme une alternative ou un complément possible aux solutions chimiques [6-16]. Ces techniques ne sont pas forcément nouvelles. À titre d'exemple, la localisation des herbicides sur le rang et le binage étaient pratiqués en Champagne ily a une trentaine d'années, dans des exploitations adeptes des semis de précision de la betterave.
Les cultures oléagineuses possèdent plusieurs atouts les prédisposant à ce type de lutte en culture : possibilité de moduler les écartements entre lignes de semis, capacité d'étouffement des mauvaises herbes (bonne capacité d'absorption d'azote pour le colza, concurrence pour la lumière pour le tournesol), faculté de compensation importante suite à des pertes de pieds occasionnelles (cas du colza), et surtout calendriers culturaux qui rendent possibles des passages d'outils en conditions météorologiques avantageuses : fin d'été pour le colza, printemps pour le tournesol et soja.

Partant de ce constat, le CETIOM a engagé au début des années 2000 un programme pluriannuel d'expérimentation de ces techniques alternatives. Les performances de diverses modalités de désherbage, associant ou non le mécanique et le chimique, ont ainsi été comparées au regard de leurs possibilités de mise en œuvre dans des conditions représentatives des grands bassins de production d'oléagineux français. Ce vaste projet d'études se poursuit à I'heure actuelle dans le cadre de partenariats régionaux ${ }^{1}$.

\section{Matériels et méthodes}

\section{Situations pédoclimatiques}

Le réseau d'essais pluriannuels rassemble une trentaine de sites mis en place pour couvrir

\footnotetext{
${ }^{1}$ À titre d'exemple, citons les activités de recherche et développement qui émanent d'une collaboration interrégionale en Bourgogne-Franche-Comté. Le projet fédère de nombreux organismes: chambres d'Agriculture, CETIOM, Arvalis-Institut du Végétal, ITB, FRCUMA, DRAF-SRPV, FREDON, INRA, Lycées agricoles de Dijon-Quétigny, Fontaines, Vesoul. Il bénéficie d'un soutien financier des Agences de I'Eau Rhône-Méditerranée-Corse, Seine-Normandie, Loire-Bretagne, du conseil régional de Bourgogne et du CASDAR.
} 
une large gamme de milieux et climats contrastés. Les sols ayant servi de supports de tests étaient variés et couvraient les textures suivantes: argilo-limoneux, argilo-limono-sableux, limons moyens, limons argileux, sablolimono-argileux et sable. Les sols argilo-calcaires superficiels ("petites terres à cailloux") étaient représentés dans quelques sites, en Côte-d'Or et Lorraine notamment. Les expérimentations en tournesol ont été réalisées entre 2000 et 2006 dans les régions Franche-Comté, Rhône-Alpes, Midi-Pyrénées et Centre. En colza, l'essentiel des références a été acquis entre 2002 et 2008 dans l'est et le nord-est de la France. Les tout premiers travaux portant sur le désherbage combiné du colza (herbicide de post-semis prélevé sur le rang puis binage) remontent à 1992, aux lendemains de la réforme de la PAC. Concernant le soja, trois essais ont été conduits dans les départements de I'Hérault et du Rhône en 2002, 2004 et 2005. Enfin, en complément de ces différents sites, le CETIOM a suivi une quinzaine de parcelles chez des agriculteurs biologiques entre 1999 et $2002^{2}$.

\section{Dispositifs, matériels et modalités testés}

Les essais étaient majoritairement implantés en bandes sans répétition, en parcelle d'agriculteur. Des témoins non désherbés, intercalés entre les modalités désherbées, ont permis d'estimer l'infestation naturelle des différents sites. Aucune exigence n'était requise vis-à-vis du type de flore et du niveau d'infestation, si bien que les espèces observées appartiennent à des familles botaniques diverses et variées, plus ou moins faciles à éliminer par voie chimique et plus ou moins concurrentielles des cultures.

Les protocoles poursuivaient deux objectifs: (i) évaluer l'efficacité et la sélectivité de plusieurs outils de désherbage mécanique en complément ou non d'un désherbage chimique, (ii) identifier, en conditions réelles, les différents facteurs expliquant la réussite et la faisabilité de ces techniques alternatives.

Les modalités mises en place ont permis d'évaluer et comparer différentes techniques de lutte chimique, mécanique, mixte, associant ou non plusieurs outils, avec des dates et des conditions d'intervention distinctes (tableau 1). Certains protocoles cherchaient à évaluer, pour des outils en particulier, l'impact de réglages ou équipements spécifiques sur l'efficacité et la sélectivité. Au cours du programme expéri-

\footnotetext{
${ }^{2}$ L'activité, menée en collaboration avec plusieurs partenaires des régions Poitou-Charentes, MidiPyrénées et Languedoc-Roussillon, avait bénéficié de soutiens financiers de I'ONIOL, I'ACTA et le ministère de l'Agriculture et de la Pêche.
}

mental, trois outils de lutte mécanique ont été étudiés :

- La bineuse : outil emblématique des cultures à écartements larges, la bineuse coupe les racines des mauvaises herbes présentes dans l'inter-rang. Son action peut, en outre, étouffer les adventices présentes sur le rang par projection de terre au pied des plantes sur le rang (fonction buttage). Lors de passage de l'outil à des stades juvéniles de la culture, des équipements spécifiques peuvent être utilisés pour protéger la plante cultivée. Plus la largeur entre les rangs augmente, plus le contrôle des mauvaises herbes, par unité de surface, sera amélioré. En fonction du stade phénologique de la culture, et du souhait ou non de rapprocher l'action mécanique près du rang cultivé, divers modèles de pièces travaillantes ont pu être testés (socs cœur, pattes d'oies, lames "lelièvre », etc.). Le programme expérimental a aussi permis de tester des bineuses qui désherbent sur le rang grâce à des éléments mécaniques spécifiques (doigts Kress, moulinets Steketee). La largeur de travail de la bineuse est toujours dépendante de celle du semoir et recouvre une distance de 6 ou 8 rangs semés. Cette largeur limitée du travail réduit notablement le débit de chantier de ce mode de désherbage.

Dans de nombreux essais, le CETIOM a testé la technique qui consiste à associer un désherbage chimique localisé sur le rang, au moment du semis, et le binage en cours de culture [810]. Un kit de désherbage localisé est, dans ce cas, disposé sur un semoir monograine. Une ou deux buses de pulvérisation sont positionnées derrière chaque élément semeur et pulvérisent sur une largeur au sol de $20 \mathrm{~cm}$. Le pourcentage de surface traitée est d'autant plus réduit que l'écartement entre rangs est élevé, ce qui rend la culture du tournesol particulièrement candidate à cette alternative de désherbage. Le kit de pulvérisation localisée est muni d'un système électronique permettant de réguler le débit de la bouillie épandue selon la vitesse d'avancement du tracteur. Dans les essais, la cuve contenant I'herbicide était positionnée au-dessus du semoir ou à l'avant du tracteur et permettait une autonomie de 7 à 8 ha pour un volume de 200 litres de bouillie appliqué à I'hectare.

- La herse étrille : bien connu des agriculteurs en mode biologique, ce matériel simple de conception est constitué de panneaux articulés et indépendants sur lesquels sont fixées des dents longues et souples, à ressort ou en arc de cercle. Cet outil polyvalent travaille sur toute la surface de la parcelle et peut donc s'utiliser sur des cultures semées à écartement réduit. Les vibrations des dents déracinent ou recouvrent de terre les jeunes pousses d'adventices. La largeur de travail fluctue entre 6 et
$24 \mathrm{~m}$. Le réglage de l'agressivité de la herse est possible en jouant sur l'inclinaison des dents, la profondeur de travail et la vitesse d'avancement.

- La houe rotative: moins connu, ce matériel traîné, simple de conception, présente une ou plusieurs séries de roues étoilées, fixées sur un axe horizontal par des bras mobiles et possédant des dents aux extrémités en forme de cuillères. En travaillant à vitesse élevée (15 à 18 km/ h), les dents frappent le sol et soulèvent les premiers centimètres de terre mettant « à nu » les adventices tout juste germés. À l'image de la herse étrille, toute la surface de la parcelle est travaillée par la houe rotative. La largeur la plus courante est de 4,70 m, mais il est possible d'aller jusqu'à $9 \mathrm{~m}$. L'agressivité de l'outil peut être modifiée en ajoutant des ressorts sur les bras mobiles ou en augmentant la pression au sol par l'ajout de masses sur le bâti de l'outil.

\section{Observations et mesures réalisées}

Les mesures de sélectivité ont été réalisées par comptage du peuplement de la culture avant et après chaque passage des outils à partir de placettes fixes repérées. Pour les passages en prélevée, les pertes de graines en cours de germination sont également estimées.

Pour la mesure de l'efficacité, deux méthodes ont été utilisées. La première impose un dénombrement des adventices avant le passage de l'outil, puis environ 7 jours après, à partir de secteurs délimités. L'autre méthode consiste à mesurer, après l'intervention, le pourcentage de recouvrement du sol par chacune des espèces ainsi que leur hauteur et à comparer cette notation à celle issue des témoins non désherbés (notation dite de « biovolume »). Quelque temps après la dernière intervention, en appliquant l'une ou l'autre méthode, un bilan de l'enherbement est réalisé pour connaître la performance globale du programme de désherbage mis en œuvre.

\section{Résultats et discussions}

\section{Sélectivité}

Les pertes de plantules cultivées causées par les techniques de lutte mécanique sont sous l'effet de plusieurs facteurs conjuguant les types d'outils, leurs réglages ainsi que le stade phénologique de la culture au moment de leur utilisation. Quel que soit le stade d'intervention, les méthodes mécaniques de désherbage «en plein » ont une sélectivité plus faible que le binage, ce dernier ne travaillant que l'interlignes (tableau 2). Les résultats d'essais attestent d'une bonne sélectivité de la herse étrille en prélevée, pour une profondeur normale de semis de colza ou soja (également vrai pour le 


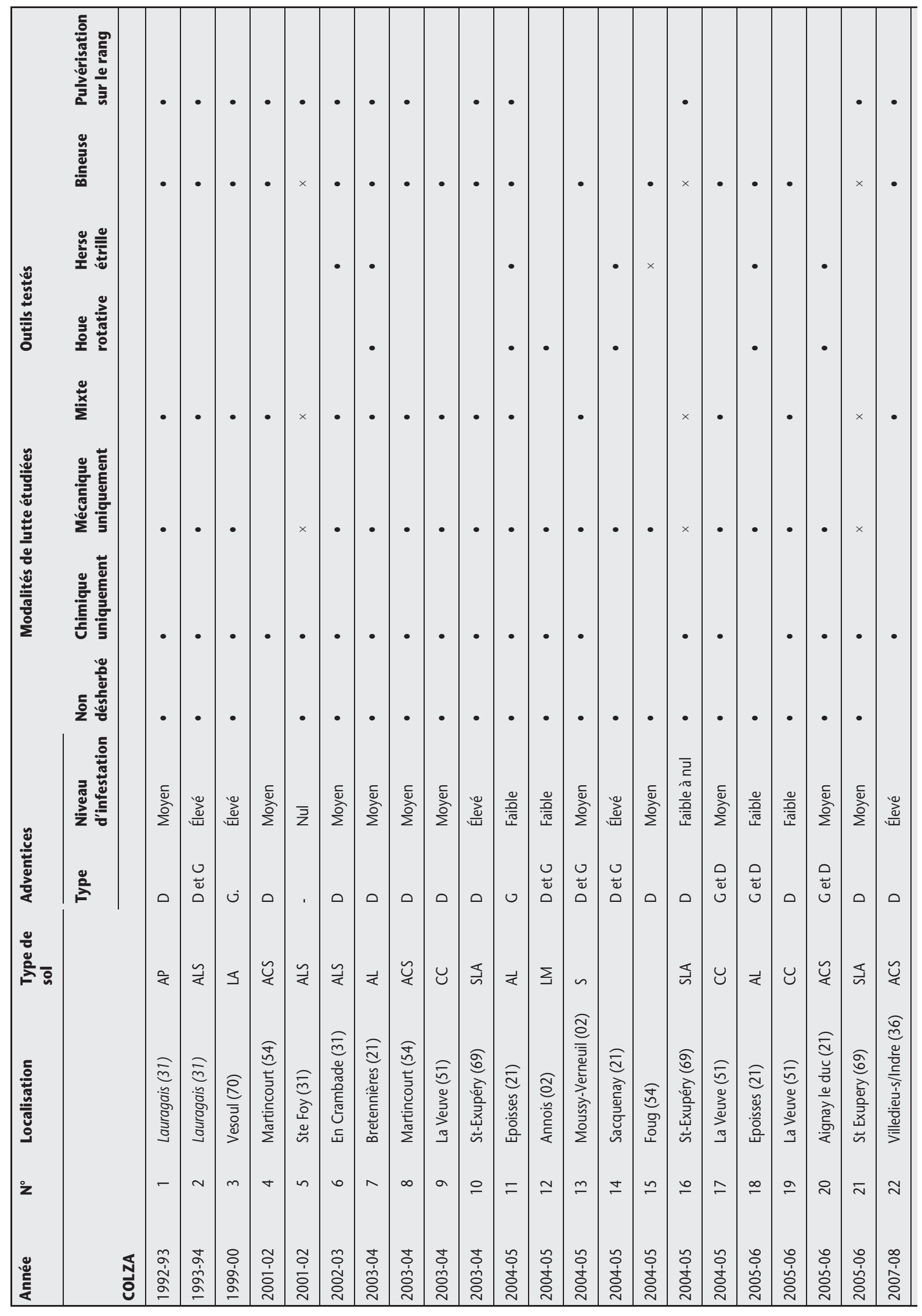

172 OCL VOL. 16 N 3 MAI-JUIN 2009 


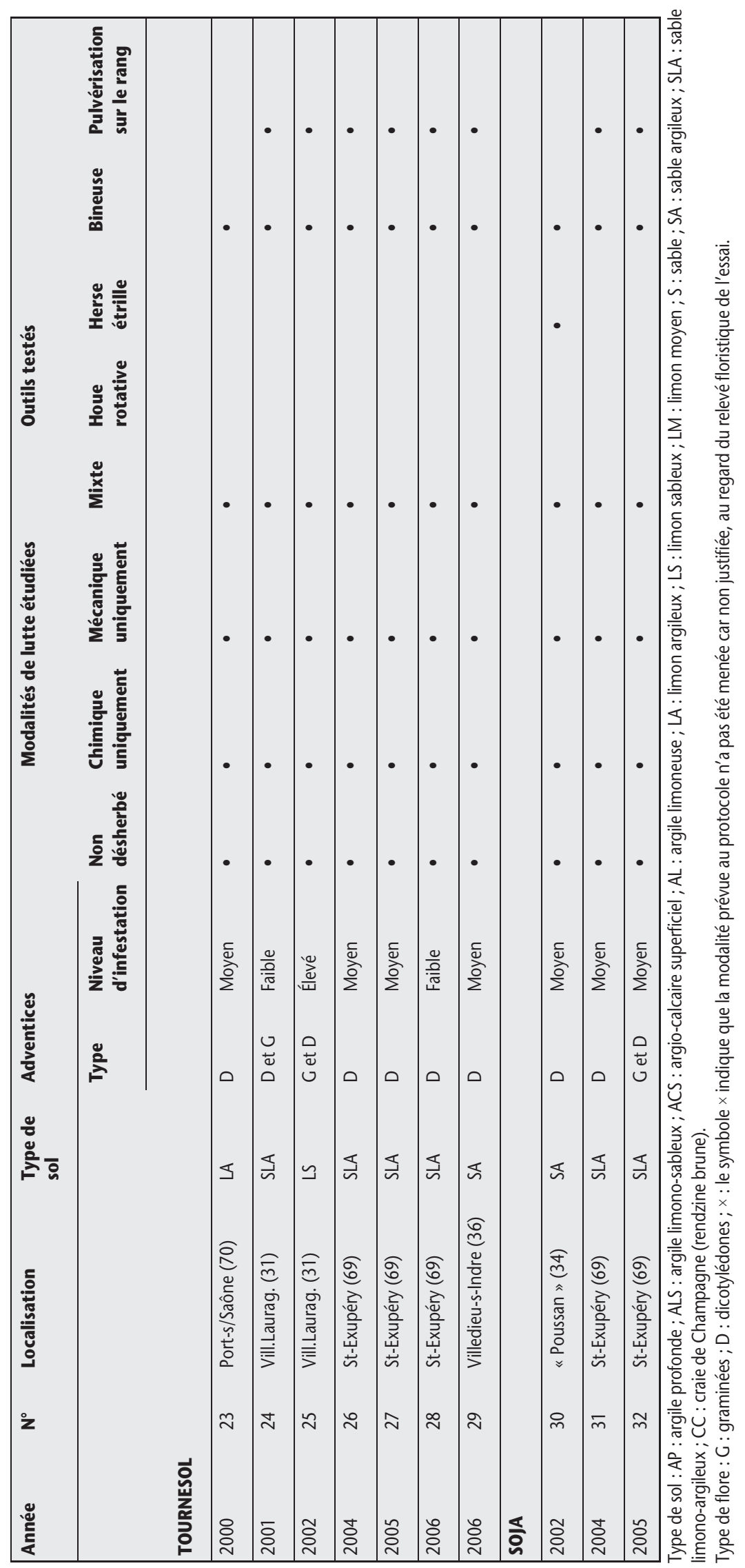


Tableau 2. Pourcentages de pertes de pieds estimés après utilisation d'outil en fonction du stade du colza.

\begin{tabular}{|c|c|c|c|c|c|c|c|}
\hline & \multicolumn{7}{|c|}{$\begin{array}{l}\text { Stade du colza } \\
\text { (code BBCH) }\end{array}$} \\
\hline & \multirow{2}{*}{$\begin{array}{l}\text { Non levé } \\
- \\
(00-08)\end{array}$} & \multirow{2}{*}{$\begin{array}{l}\text { Cotylédon } \\
\text { A } \\
(09-10)\end{array}$} & \multirow{2}{*}{$\begin{array}{l}2 \text { feuilles } \\
\text { B2 } \\
(12)\end{array}$} & \multirow{2}{*}{$\begin{array}{l}4 \text { feuilles } \\
\text { B4 } \\
(14)\end{array}$} & \multirow{2}{*}{$\begin{array}{l}\text { 6 feuilles } \\
\text { B6 } \\
(16)\end{array}$} & \multicolumn{2}{|c|}{ Reprise de végétation } \\
\hline & & & & & & $\begin{array}{l}\text { C1 } \\
\text { (31) }\end{array}$ & $\begin{array}{l}\text { C2 } \\
\text { (32) }\end{array}$ \\
\hline Houe rotative & $5 \%( \pm 5)$ & $5 \%( \pm 7)$ & $4 \%( \pm 4)$ & $5 \%( \pm 5)$ & $5 \%( \pm 5)$ & non testé & non testé \\
\hline Herse étrille & $8 \%( \pm 8)$ & $29 \%( \pm 9)$ & $17 \%( \pm 3)$ & $8 \%( \pm 3)$ & $8 \%( \pm 3)$ & $8 \%( \pm 3)$ & $8 \%( \pm 3)$ \\
\hline Bineuse & non testé & non testé & $12 \%$ & $1 \%( \pm 1)$ & $1 \%( \pm 1)$ & $1 \%( \pm 1)$ & $1 \%( \pm 1)$ \\
\hline
\end{tabular}

tournesol, d'après les enquêtes auprès d'agriculteurs bio). En revanche, le hersage de postlevée précoce aux premiers stades de la culture peut s'accompagner de dégâts sérieux (casses de germes ou déchaussements) pouvant altérer le potentiel de rendement. Les pertes ont été observées de la levée aux stades trois à quatre feuilles pour le colza (jusqu'à $40 \%$ de pertes

constatées), de la levée jusqu'à deux feuilles unifoliées du soja et de la levée jusqu'à une paire de feuilles du tournesol. Ces défauts de sélectivité proscrivent donc l'utilisation de la herse à des stades juvéniles de ces trois cultures. Pour un usage un peu plus tardif, les pertes de pieds mesurées fluctuent entre 5 et $10 \%$. Les réglages de l'appareil sont également

Tableau 3. Créneaux d'utilisation des outils mécaniques en fonction des cultures et de leur stade de développement.

\begin{tabular}{|c|c|c|c|}
\hline & Herse étrille & Houe rotative & Bineuse \\
\hline \multicolumn{4}{|l|}{ Colza } \\
\hline Germination (00-08) & ** & ** & 0 \\
\hline Cotylédon A1 (09-10) & 0 & ** & 0 \\
\hline B1 (11) & 0 & ** & * \\
\hline B2 (12) & 0 & ** & ** \\
\hline B3 (13) & * & ** & $\star *$ \\
\hline B4 à C2 (14 à 32) & ** & ** & $* \star *$ \\
\hline \multicolumn{4}{|l|}{ Tournesol } \\
\hline Germination (00-08) & * & * & 0 \\
\hline Cotylédon A2 (09-10) & 0 & ** & 0 \\
\hline B1-B2 (12) & * & ** & ** \\
\hline B3-B4 (14) & ** & ** & $* * *$ \\
\hline B5 à E1 (15-51) & ** & Non testé & $* * *$ \\
\hline \multicolumn{4}{|l|}{ Soja } \\
\hline Germination (05) & ** & 0 & 0 \\
\hline Cotylédon A2 (09-10) & 0 & ** & 0 \\
\hline V1 (12) & ** & ** & 0 \\
\hline V2 (13) & ** & ** & ** \\
\hline V3-V4 (14-15) & ** & Non testé & $* * *$ \\
\hline R1 - R3 (60-65) & 0 & Non testé & $* * *$ \\
\hline
\end{tabular}

0 utilisation non recommandée ; * utilisation possible, risque de pertes d'environ $10 \%$; ** utilisation possible (avec protèges-plants pour la bineuse), pertes insignifiantes $<5 \%$; ${ }^{* * *}$ utilisation possible, effet buttage sur le rang (absence de protèges-plants). déterminants. Ainsi, à vitesse d'étrillage élevée (7 à $10 \mathrm{~km} / \mathrm{h}$ ), l'outil provoque plus de pertes lorsque les plantes sont jeunes. Pour supporter les dommages, le colza peut naturellement mettre à profit ses facultés de compensation grâce à ses ramifications. Pour le tournesol et le soja, il est souhaitable d'anticiper la prévision des pertes en augmentant la densité de semis d'environ $10 \%$ par rapport aux recommandations habituelles.

Les tests de houe rotative réalisés à haute vitesse (12 à $18 \mathrm{~km} / \mathrm{h}$ ) sur colza entre la levée et le stade 6 feuilles ont donné pleine satisfaction en termes de sélectivité : les pertes sont inférieures à $5 \%$ dans 3 essais sur 4 et ne dépassent jamais $10 \%$. À la différence de la herse étrille, la houe rotative peut donc s'utiliser en colza sur un créneau continu, sans risques irréversibles pour la croissance et l'élaboration du rendement.

Les binages de colza ont généralement été expérimentés à l'automne au stade 6 à 8 feuilles vraies. Globalement, dans ces conditions et avec des réglages de matériel à l'optimum, la sélectivité est quasi parfaite. En tournesol et soja, les binages ont été testés respectivement à partir de 3 paires de feuilles et deuxième feuille trifoliée jusqu'au stade limite de passage de l'outil. Dès lors que les matériels de semis et de binage sont réglés harmonieusement (nombre de rangs, écartement, largeur de voie de tracteur) et utilisés à une vitesse adaptée aux conditions, les pertes sont minimes et sans conséquence néfaste pour le potentiel des cultures.

\section{Efficacité vis-à-vis des adventices}

\section{Efficacité selon les stades de développement}

En moyenne, I'efficacité directe obtenue à partir des méthodes mécaniques en plein n'est jamais très élevée, et en rien comparable à celle obtenue avec des herbicides efficaces. Pour la herse et $a$ fortiori pour la houe, la réussite est largement tributaire du développement des adventices. Dès le stade 3 feuilles, les mauvaises herbes font preuve d'une forte résistance à l'action de ces outils, au point de réduire consi- 


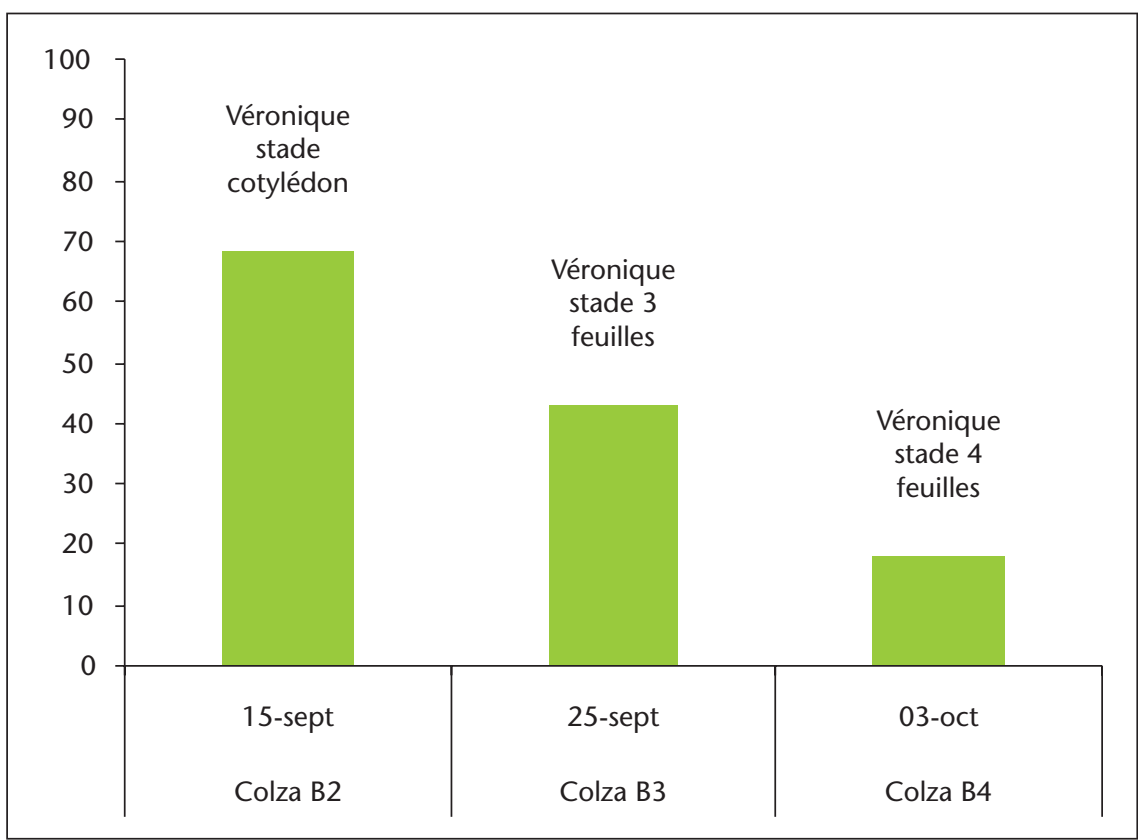

Figure 2. Efficacité (en \% par rapport au témoin non désherbé) de la houe rotative selon le stade de l'adventice, Veronica persica (colza, site $\left.n^{\circ} 14\right)$

dérablement l'intérêt de leur utilisation (figure 2).

Pour la houe rotative, l'optimum pour espérer au mieux $90 \%$ d'élimination se situe entre les stades « fil blanc » (tout début de la germination) et une feuille déployée des mauvaises herbes. Cet intervalle de temps, très court, nécessite un suivi quasi quotidien de l'évolution de l'enherbement lorsque les conditions météo et d'humidité de sol sont réunies pour toute intervention. Un passage unique de houe, même s'il est réalisé dans de bonnes conditions, est généralement insuffisant pour assurer un contrôle efficace et durable de l'enherbement. En effet, un grand nombre d'espèces adventices présentent une levée échelonnée dans le temps, si bien que seuls des passages répétés ou combinés avec un désherbant au préalable voire avec un autre outil mécanique assurent un contrôle acceptable (figure $3 A$ ).

L'efficacité de la herse reste en tendance supérieure à celle de la houe, pour une adventice donnée, à un stade identique. Grâce à un travail plus profond et plus agressif, les performances du hersage peuvent atteindre 90 à $95 \%$ d'efficacité sur des plantules à moins de 4 feuilles (figure 4). L'avantage que présente la herse est de pouvoir se permettre d'attendre une levée plus « franche » des mauvaises herbes et d'intervenir sur un colza ou un soja déjà bien enracinés. Ces derniers, bien implantés, supportent aisément un réglage agressif de l'appareil (vitesse plus élevée et dents moins inclinées) qui améliore d'autant l'efficacité du désherbage. Dans des situations de flore peu com- localisée d'herbicide sur la ligne au moment du semis et un binage en complément semble particulièrement prometteuse. De nombreuses références attestent d'efficacités satisfaisantes et souvent comparables aux programmes chimiques classiques appliqués sur toute la surface (figure 3C et 3D). Si l'on cherche à renoncer aux herbicides, un binage précoce relayé par un second semble être un minimum, notamment pour des cultures à faible pouvoir concurrentiel en début de cycle (cas du tournesol et du soja notamment).

\section{Efficacité selon la nature des adventices}

Les résultats d'essais ont montré des efficacités contrastées selon les espèces de mauvaises herbes et les familles d'outils testées. Ainsi, une espèce profondément et solidement enracinée dès ses premiers stades est plus difficile à détruire qu'une mauvaise herbe plus superficiellement enracinée. L'appareil végétatif (aérien et souterrain) prédispose certaines espèces à supporter les blessures, arrachements ou enfouissements et à résister au dessèchement dans les jours qui suivent l'intervention. Chez le colza, le bleuet (Centaurea cyanus) et le vulpin des champs (Alopecurus myosuroides) sont par exemple plus difficiles à détruire car mieux enracinés que la pensée des champs (Viola arvensis), la capselle bourseà-pasteur (Capsella bursa pastoris) ou les géraniums (Geranium sp.). Chez le tournesol ou le soja, le chénopode blanc (Chenopodium album), la mercuriale annuelle (Mercurialis annua) ou l'amarante réfléchie (Amaranthus retroflexus) sont plus vulnérables que la lampourde à gros fruits (Xanthium strumarium), le datura stramoine (Datura stramonium), la renouée persicaire (Polygonum persicaria) et les graminées estivales telles que panic piedde-coq (Echinochloa crus-galli) ou sétaires (Setaria sp.). Les essais ont montré que tous les outils signent une faiblesse d'efficacité en particulier sur les graminées et repousses de céréales.

\section{Efficacité selon les caractéristiques pédoclimatiques}

Humidité et prévisions météo : très tôt après une pluie, dès que la terre n'adhère plus aux roues du tracteur, le passage de houe peut être envisagé alors que celui de la herse nécessite un sol frais et celui de la bineuse un sol ressuyé. À l'inverse, sur sol sec, la houe fonctionne moins bien que la herse et la bineuse (tableau 3). La décision de passer les outils mécaniques doit être guidée par des prévisions météorologiques favorables les jours suivant l'intervention, à savoir des conditions séchantes permettant la dessiccation des mauvaises herbes extirpées ou déchaussées. Dans le cas contraire, nous constatons souvent des effets inverses à ceux escomptés : repiquage ou sti- 


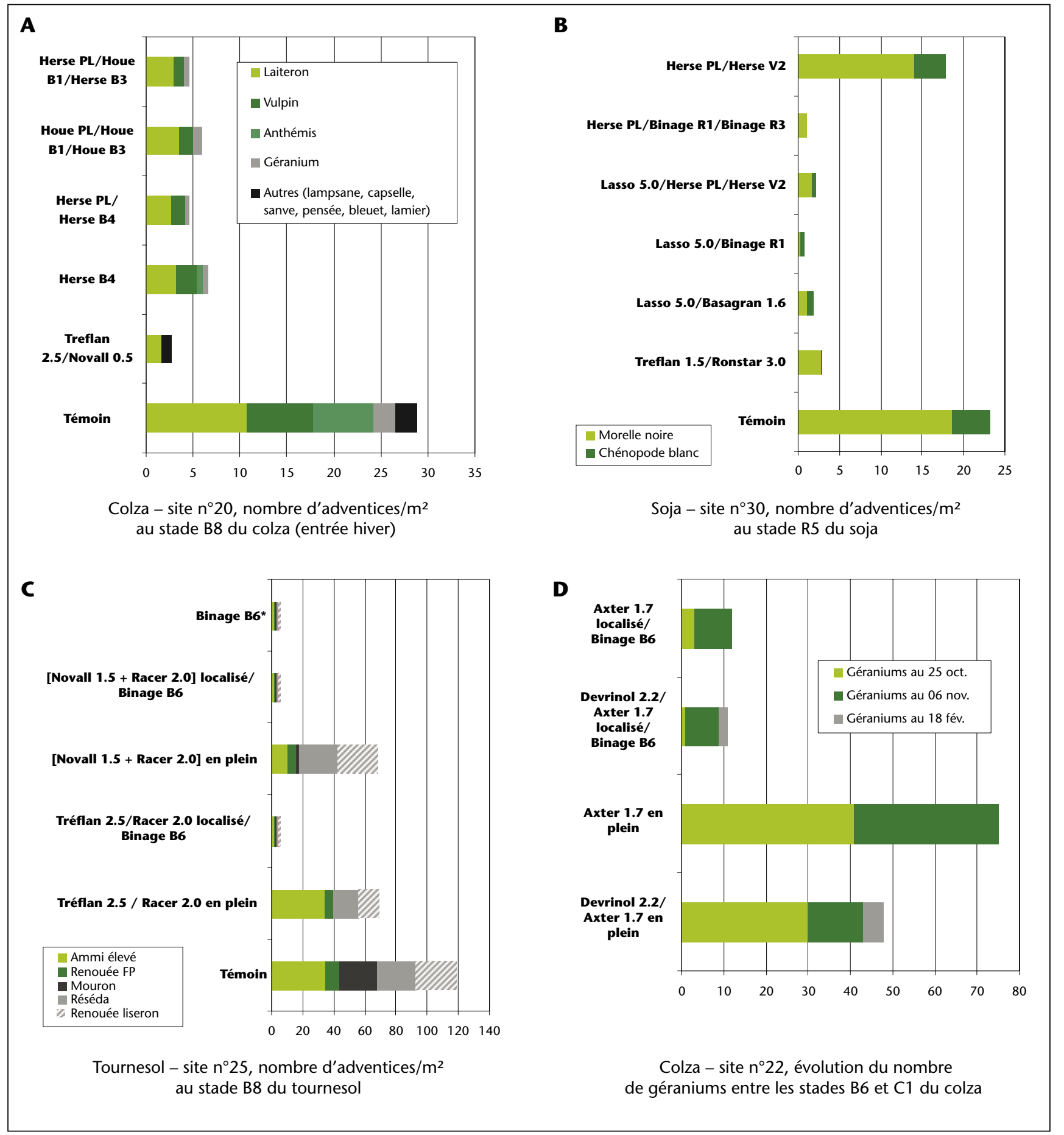

Figure 3. Intérêt des associations de techniques chimiques et mécaniques

* Le comptage est réalisé sur l'inter-rang uniquement.

mulation de la germination après émiettement et remuement de la surface du sol.

Texture, structure, charge en cailloux : les passages d'outils mécaniques étaient facilités sur des terrains à texture légère (tableau 4). Ils ont procuré des résultats tout à fait satisfaisants sur sol argilo-calcaire, en présence de petites mottes de terre. Les sols à cailloux ne sont pas rédhibitoires mais ils provoquent une usure plus rapide des matériels (socs de bineuse et extrémités des dents de houe rotative). En sol hydromorphe lourd, à forte teneur en argile, la durée du temps de ressuyage après une pluie réduit considérablement les chances d'intervenir à l'optimum, en raison d'un créneau resserré d'utilisation des outils. Sur sol limoneux battant, la herse étrille et la houe rotative sont délicates à utiliser. En conditions trop humides, les 


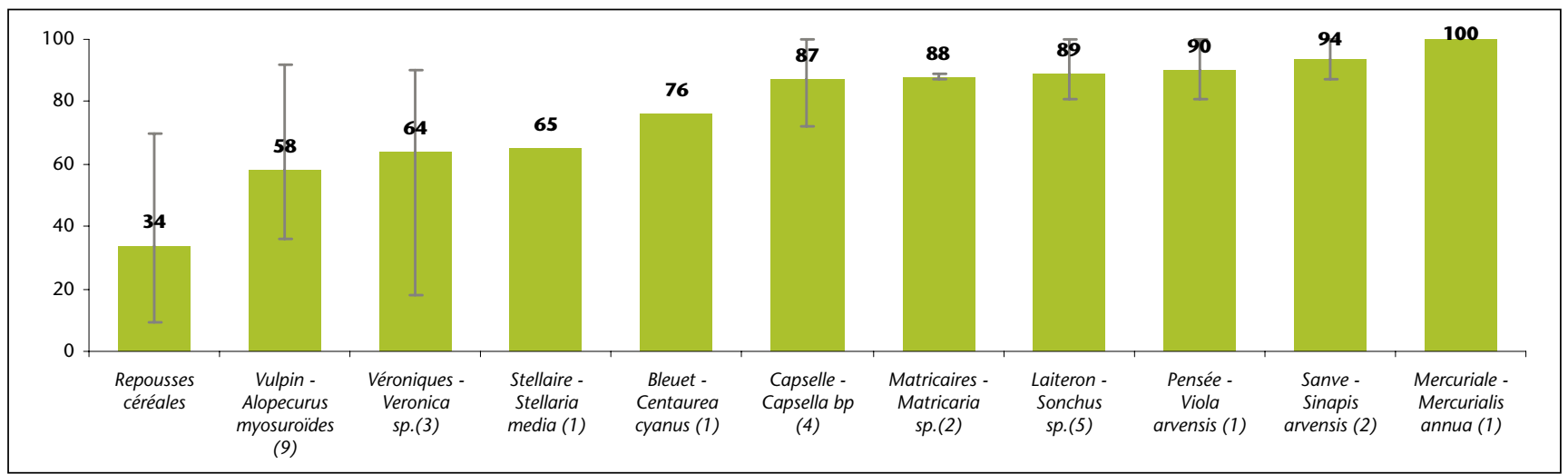

Figure 4. Synthèse des efficacités (en \% par rapport au témoin non désherbé) de la herse étrille sur les adventices du colza - les chiffres entre parenthèses indiquent le nombre de références.

Tableau 4. Adaptations des outils aux types de sols (d'après Groupe Inter-régional Bourgogne Franche-Comté).

\begin{tabular}{|llll|}
\hline & Herse étrille & Houe rotative & Bineuse \\
\hline Sables & $* *$ & 0 & $* *$ \\
\hline Limons sains & 0 & $* *$ & $*$ \\
\hline Limons battants & 00 & $* *(1)$ & $* *$ \\
\hline Argilo-limoneux & $* *$ & $* *$ & $*$ \\
\hline Argileux & 0 & $*$ & $* *(2)$ \\
\hline Petites terres à cailloux & $* *$ & $* *$ & $*$ * \\
\hline
\end{tabular}

00 utilisation à proscrire ; 0 utilisation déconseillée ; * utilisation possible avec réglages précis ; ** utilisation recommandée.

(1) Fonction « écroûtage ».

(2) En fonction de la charge en cailloux.

particules de terre s'agglomèrent autour des dents et réduisent leur pénétration dans le sol. En conditions sèches, herse et houe ne peuvent briser la croûte de battance et agissent trop superficiellement. Dans ce cas de figure, la bineuse soulève la première épaisseur de sol avec plus de risques d'altérer l'enracinement de la culture que de chances de nuire aux mauvaises herbes.
Nivellement et gestion des résidus en surface : ils contribuent notamment à la sélectivité et à l'efficacité de la herse étrille. Un hersage de qualité s'obtient à partir d'un travail horizontal et parallèle au sol et nécessite donc, en amont, une préparation très soignée de l'horizon superficiel. Cette préparation doit intégrer des objectifs de gestion des pailles pour éviter les phénomènes de bourrages qui gênent l'action des dents.

\section{Évaluation économique et environnementale}

\section{Des économies sur le poste herbicide}

L'analyse économique a été effectuée à partir $d$ 'hypothèses de calculs et d'itinéraires types (tableaux 5 et 6). Les opérations s'effectuent à partir des coûts liés (i) aux herbicides, (ii) aux passages du pulvérisateur (amortissement du matériel + coût de traction), (iii) aux passages d'outils mécaniques (amortissement du matériel utilisé + coût de traction) et (iv) au coût de la main-d'œuvre salariale (temps de travail $\times$ coût horaire). Signalons la prise en compte d'une hypothèse haute de coût d'amortissement $d u$ kit de pulvérisation : $3600 €$ amortis sur 7 ans, sur la base de 60 ha déployés/an. Cette charge fixe peut être réduite en amortissant l'équipement spécifique sur une plus grande surface. L'économie faite sur les herbicides (55 à $66 \%$ de quantité en moins selon l'écartement) n'est pas totalement contrebalancée par le surcoût lié aux matériels (kit + bineuse) et les écarts entre les deux techniques oscillent entre 5 et 15 euros/ha, en faveur du désherbage mixte. En revanche, le binage est consommateur de temps à I'hectare: environ 5 à 6 fois plus qu'une application chimique. II implique donc

Tableau 5. Hypothèses de calculs retenues dans le cadre de l'étude économique (sources : index des prix et normes agricoles 2006-07, chambre d'agriculture 89).

\begin{tabular}{|c|c|c|c|c|c|}
\hline Matériel & $\begin{array}{l}\text { Prix achat } \\
(€ \mathrm{HT})^{*}\end{array}$ & $\begin{array}{l}\text { Surface travaillée } \\
\text { (ha/an) }\end{array}$ & $\begin{array}{l}\text { Débit de chantier } \\
\text { (ha/h) }\end{array}$ & $\begin{array}{l}\text { Puissance traction } \\
\text { (CV) }\end{array}$ & $\begin{array}{l}\text { Coût total estimé } \\
(€ / \text { ha })\end{array}$ \\
\hline Pulvérisateur 24 m, 1500 litres & 15000 & 600 & 10 & 100 & 6,2 \\
\hline Kit de pulvérisation localisée & 3600 & 60 & - & - & 8,6 \\
\hline Herse étrille $12 \mathrm{~m}$ & 7000 & 140 & 9,6 & 100 & 9,8 \\
\hline Houe rotative $4,7 \mathrm{~m}$ & 9700 & 200 & 5 & 80 & 11,8 \\
\hline Bineuse à dents -6 rangs & 7000 & 60 & 1,8 & 100 & 31,1 \\
\hline
\end{tabular}

* La durée d'amortissement retenue s'élève à 7 ans ; SMIC : 12,99€ HT/h. 
Tableau 6. Comparaisons de coûts économiques et temps de travaux de différents itinéraires de désherbage, à partir de cas-types en colza et tournesol.

\begin{tabular}{|c|c|c|c|c|c|c|}
\hline \multirow[t]{2}{*}{ Type de programme de désherbage } & \multicolumn{5}{|c|}{ Coût moyen $€ /$ ha } & \multirow{2}{*}{$\begin{array}{l}\text { Temps de } \\
\text { travail moyen } \\
\text { (min/ha) }\end{array}$} \\
\hline & Herbicide & Pulvérisateur & $\begin{array}{l}\text { Outils } \\
\text { mécaniques }\end{array}$ & $\begin{array}{l}\text { Main- } \\
\text { d'œuvre }\end{array}$ & Total & \\
\hline \multicolumn{7}{|l|}{ COLZA } \\
\hline \multicolumn{7}{|c|}{ Cas 1 : flore difficile à contrôler (géranium tige grêle par exemple) } \\
\hline Devrinol 1.3 en plein // Colzor trio 3.5 en plein // - & 100 & 10 & 6 & 7 & 123 & 37 \\
\hline Devrinol 1.3 en plein // Colzor trio 3.5 localisé // binage & 57 & 5 & 39 & 14 & 115 & 70 \\
\hline Devrinol 1.5 en plein // - // herse étrille puis binage & 32 & 5 & 39 & 14 & 90 & 71 \\
\hline \multicolumn{7}{|c|}{ Cas 2 : pression moyenne (capselle, matricaire, stellaire par exemple) } \\
\hline - // Novall 2.5 en plein // - & 88 & 5 & - & 1 & 94 & 6 \\
\hline - // Novall 2.5 localisé // binage & 35 & - & 33 & 8 & 76 & 39 \\
\hline - // Novall 1.5 en plein // houe rotative $\times 2$ & 53 & 5 & 19 & 6 & 82 & 30 \\
\hline - // Novall 1.5 en plein // herse étrille $\times 2$ & 53 & 5 & 17 & 4 & 78 & 19 \\
\hline \multicolumn{7}{|c|}{ Cas 3 : pression faible (capselle, alchémille, lamiers par exemple) } \\
\hline - // Butisan S 2.0 en plein // - & 68 & 5 & - & 1 & 74 & 6 \\
\hline - // Butisan S 2.0 localisé // binage & 27 & - & 33 & 8 & 68 & 39 \\
\hline - // Butisan S 2.0 localisé // houe rotative $\times 2$ & 27 & - & 19 & 6 & 52 & 30 \\
\hline - // Butisan S 2.0 localisé // herse étrille & 27 & - & 17 & 3 & 47 & 13 \\
\hline \multicolumn{7}{|l|}{ TOURNESOL } \\
\hline \multicolumn{7}{|c|}{ Cas 1 : flore difficile (renouées, crucifères, graminées par exemple) } \\
\hline Prowl 4002.5 // Challenge 6003.5 en plein // - & 110 & 10 & 6 & 7 & 133 & 37 \\
\hline Prowl 4002.5 // Challenge 6003.5 localisé // binage × 2 & 60 & 5 & 63 & 21 & 149 & 104 \\
\hline Prowl 4002.5 // Challenge 6003.5 localisé // binage & 61 & 5 & 39 & 14 & 119 & 70 \\
\hline \multicolumn{7}{|c|}{ Cas 2 : pression moyenne (capselle, chénopodes, mercuriale, morelle par exemple) } \\
\hline Mercantor Gold 1.4 // Ronstar 2.0 en plein // - & 75 & 10 & 6 & 7 & 98 & 37 \\
\hline - // Racer 2.5 en plein // - & 75 & 5 & - & 1 & 81 & 6 \\
\hline - // Racer 2.5 localisé // binage & 30 & - & 33 & 8 & 71 & 39 \\
\hline Mercantor Gold 1.4 // - // binage & 28 & 5 & 30 & 13 & 76 & 64 \\
\hline \multicolumn{7}{|c|}{ Cas 3 : pression faible (morelle, mouron des champs par exemple) } \\
\hline - // Novall 1.5 en plein // - & 53 & 5 & - & 1 & 59 & 6 \\
\hline - // Novall 1.5 localisé // binage & 18 & - & 33 & 8 & 58 & 39 \\
\hline$-/ /-/ /$ binage $\times 2$ & - & - & 49 & 13 & 62 & 67 \\
\hline - // - // herse étrille puis binage & - & - & 33 & 8 & 41 & 40 \\
\hline
\end{tabular}

Lecture : Devrinol 1.5 // - // herse étrille puis binage = Devrinol 1.5 en présemis, rien en post-semis prélevée, herse étrille et binage en post-levée

un coût de main-d'œuvre supplémentaire, évalué, dans notre simulation, sur la base du SMIC horaire. En conséquence, deux passages d'une bineuse non amortie engendrent des coûts élevés à l'hectare, notamment s'ils s'effectuent sur une surface limitée. L'utilisation de la herse étrille ou de la houe rotative est plus avantageuse du fait du débit de chantier plus élevé et de l'économie réalisée sur un désherbant de post-semis prélevée.
Les résultats des simulations démontrent globalement que les itinéraires intégrés de désherbage procurent des coûts proches ou inférieurs aux références $100 \%$ chimiques. Les chiffres demeurent toutefois indicatifs, compte tenu de 


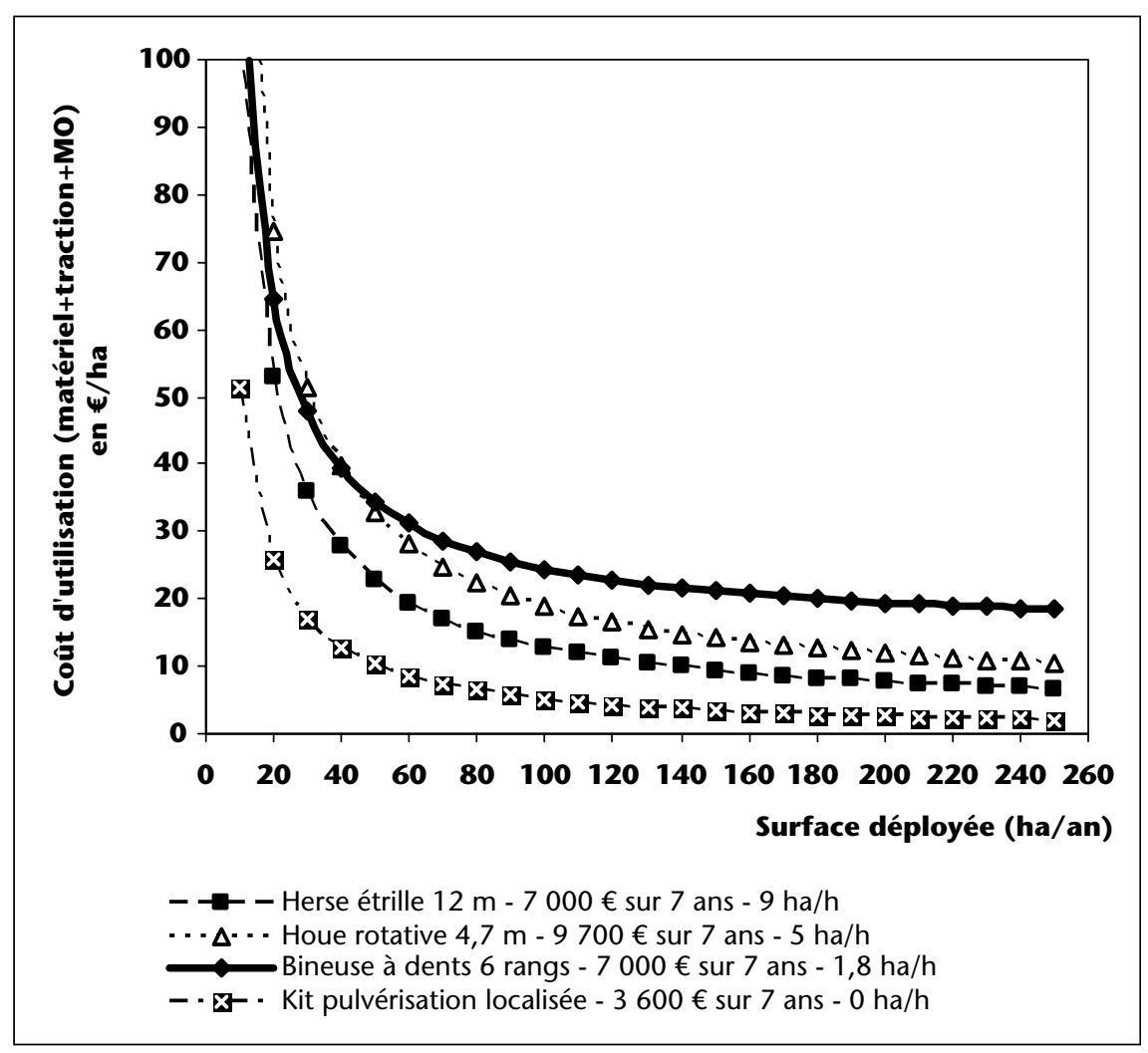

Figure 5. Synthèse des efficacités (en \% par rapport au témoin non désherbé) de la herse étrille sur les adventices du colza - les chiffres entre parenthèses indiquent le nombre de références.

la grande variation de coûts d'utilisation du matériel en fonction de la surface travaillée par an et par exploitation (figure 5). Si les matériels de désherbage mécanique peuvent être amortis sur de grandes surfaces, leur utilisation est rentable. Les techniques de désherbage intégré qui limitent I'application de produits chimiques peuvent donc permettre des économies importantes de trésorerie, à condition d'être étendues à plusieurs cultures de l'exploitation et de disposer d'un calendrier de travail et de ressources en main-d'œuvre adaptés aux besoins.

\section{Une diminution de la pression herbicide}

À partir de simulations d'itinéraires de désherbage, I'impact environnemental a été quantifié (i) en calculant les quantités de matières actives appliquées pour chaque modalité et (ii) en utilisant I'IFT, indice de fréquence de traitement (tableau 7). Cet indice est aujourd'hui utilisé, à titre de référence, notamment par les agriculteurs engagés dans des démarches de réduction des intrants pour satisfaire les exigences de la Directice-Cadre Eau. II permet d'évaluer la «pression phytosanitaire » exercée sur chaque parcelle. L'IFT herbicide est exprimé en nombre de doses homologuées d'herbicides par hectare appliquées sur la parcelle pendant che visant à caractériser, à l'échelle de la campagne agricole, les impacts sur les ressources environnementales (figure 6). Dans notre simulation (tableau 8), nous avons fait varier (i) les modalités de travail du sol en interculture labour vs non-labour ; (ii) les modalités d'application d'herbicides et (iii) les modalités de passages d'outils mécaniques en culture. Les paramètres et les méthodes de calculs reposent sur I'outil BILEO ${ }^{3}$, mis au point par le CETIOM. Les résultats montrent que l'insertion d'outils mécaniques dans les itinéraires techniques (cas-types $n^{\circ} 2,3,5$ et 6 ) ne dégrade pas les bilans énergétiques, compte tenu des hypothèses retenues. Dans les exemples considérés, 1 à 3 passages d'outils mécaniques représentent au maximum 3 à $5 \%$ du total de l'énergie fossile mobilisée pour la conduite du colza, la majeure partie des coûts énergétiques étant liée aux éléments fertilisants $(N, P, K)$. Les coûts des travaux du sol dépendent quant à eux du type de sol et de la catégorie d'outil considérée. Plus le travail est profond, plus la demande en énergie sera importante. Ainsi, dans les situations non labourées, l'agriculteur parvient à limiter sa consommation en carburant. Toutefois, le recours au glyphosate en interculture contrebalance ce bénéfice et rééquilibre le bilan environnemental, en raison des valeurs inhérentes au produit élevées (coût de la formulation, de l'emballage et de l'approvisionnement). Dans tous les cas, il semble plus pertinent de caractériser les performances énergétiques de pratiques agricoles à l'échelle du système de cultures. Dans le cadre d'une lutte intégrée contre les adventices, les successions culturales et le travail du sol sont réputés être des leviers agronomiques puissants [1, 2]. Si l'on cherche à concilier une gestion durable des adventices et une maîtrise des consommations énergétiques, une attention toute particulière doit être portée sur les cultures légumineuses a fortiori semées au printemps [18], ainsi que sur des recours uniquement occasionnels au labour et au glyphosate.

\section{Conclusion}

Ce programme d'étude a constitué une première étape dans l'acquisition de références sur les techniques de lutte mécanique associée ou non aux herbicides en oléagineux. À l'échelle de la campagne agricole, les évaluations agronomiques, environnementales et économiques des méthodes mécaniques tes-

\footnotetext{
${ }^{3}$ Inscrit dans le cadre de la Démarche de Progrès initiée par l'ONIDOL, le calculateur BILEO estime le rendement énergétique d'une parcelle de colza destinée à la production de biodiesel. L'outil intègre les coûts énergétiques de l'ensemble de l'itinéraire technique. Accessible sur www.cetiom.fr
} 
Tableau 7. Quantités de matières actives appliquées et IFT herbicide de différents itinéraires de désherbage d'oléagineux.

\begin{tabular}{|c|c|c|c|c|}
\hline \multirow{2}{*}{\begin{tabular}{|l} 
Exemples de programme de désherbage \\
COLZA
\end{tabular}} & \multicolumn{3}{|c|}{ Quantité de m.a./ha } & \multirow[t]{2}{*}{ IFT $_{\text {herbicide }}$} \\
\hline & Présemis & Prélevée & Total & \\
\hline Colzamid 1.3// Colzor trio 3.5 en plein & 585 & 1418 & 2003 & 1.34 \\
\hline Colzamid 1.3 // Colzor trio 3.5 localisé* // bineuse & 585 & 567 & 1152 & 0.81 \\
\hline Colzamid 1.5 // outils mécanique & 675 & & 675 & 0.54 \\
\hline - // Colzor trio 3.5 en plein & - & 1418 & 1418 & 0.88 \\
\hline - // Colzor trio 3.5 localisé // bineuse & - & 567 & 567 & 0.35 \\
\hline - // Novall 2.5 en plein & - & 1250 & 1250 & 1.00 \\
\hline - // Novall 2.5 localisé* // bineuse & - & 500 & 500 & 0.40 \\
\hline - // Novall 1.5 en plein // outils mécaniques & - & 750 & 750 & 0.60 \\
\hline \multicolumn{5}{|l|}{ TOURNESOL } \\
\hline Prowl 4002.5 // Challenge 6003.5 en plein // - & 1000 & 1736 & 2736 & 1.54 \\
\hline Prowl 4002.5 // Challenge 6003.5 localisé** // bineuse & 1000 & 579 & 1579 & 1.02 \\
\hline Prowl 4002.5 // herse étrille ou bineuse & 1000 & - & 1000 & 0.76 \\
\hline - // Challenge 6003.5 localisé** // bineuse & - & 579 & 579 & 0.26 \\
\hline - // Racer 2.5 en plein // - & - & 625 & 625 & 0.83 \\
\hline - // Racer 2.5 localisé $* * / /$ binage & - & 208 & 208 & 0.28 \\
\hline \multicolumn{5}{|l|}{ SOJA } \\
\hline Mercantor Gold 1.4 // Ronstar 2 & 1344 & 508 & 1852 & 1.67 \\
\hline - // Ronstar 3 localisé* // herse étrille et/ou bineuse & - & 305 & 305 & 0.40 \\
\hline - // Afalon L 1 localisé* // herse étrille et/ou bineuse & - & 180 & 180 & 0.19 \\
\hline
\end{tabular}

* En colza et soja, le kit de localisation de l'herbicide permet une application sur $20 \mathrm{~cm}$ pour un inter-rang de $50 \mathrm{~cm}$, dans cet exemple, soit une réduction de $60 \%$ des quantités.

** En tournesol, le kit de localisation permet une réduction de $65 \%$ des quantités.

tées indiquent des performances intéressantes, notamment en association avec d'autres méthodes culturales ou prophylactiques. Pourtant, d'après les dernières enquêtes menées par le CETIOM, les surfaces actuelles concernées par de telles stratégies (mécaniques seules ou en complément du chimique) sont relativement minimes : notamment pour le colza, la culture phare de la filière oléagineuse (2\% des surfaces). Dans certaines régions traditionnelles de production de tournesol (Midi-Pyrénées et Poitou-Charentes par exemple), le binage se pratique couramment. Quasiment un hectare sur deux est concerné. Des entretiens auprès $d^{\prime}$ agriculteurs ont permis $d^{\prime}$ 'identifier les freins au développement de la lutte mécanique en culture. Le besoin de changer d'état d'esprit, les contraintes d'organisation du travail et les investissements nécessaires sont apparus comme les principaux points de blocage. Dans un contexte agricole où la taille moyenne des exploitations va grandissante et où les ressources en main-d'œuvre sont de plus en plus limitées, seule la disponibilité sur le marché de l'agroéquipement d'outils mécaniques perfectionnés en matière de guidage et de débit de chantier peut permettre le développement significatif des techniques alternatives aux herbicides. Certains constructeurs de bineuses proposent aujourd'hui des systèmes de guidage mécanique (coutres circulaires marquant le sol, ou autopilotage électrohydraulique avec roues palpeuses), voire des guidages automatiques basés sur la détection de la culture grâce à un GPS ou une caméra. Ces systèmes sophistiqués gagneront à être connus par les producteurs de grandes cultures oléagineuses. Enfin, il faut signaler que l'arrivée récente sur le marché de nouveaux herbicides de post-levée du tournesol devrait ouvrir de nouvelles perspectives de réduction des risques de pollution des eaux. En effet, ces interventions ne nécessitent que de faibles quantités et sont basées sur l'observation de la flore présente dans la parcelle.

Remerciements. Le CETIOM remercie l'ensemble des partenaires qui ont contribué à l'acquisition des données : le Lycée agricole de Vesoul, les Chambres d'agriculture 02, 21, 32, 51, 54, I'INRA de Dijon, le CREAS de StExupéry, I'ITB, la FREDON 54, Agro-transfert Ressources et Territoires, et les organismes partenaires du Groupe Inter-Régional de Bourgogne Franche-Comté. Actuel partenaire du projet intitulé "Optimiser et promouvoir le désherbage mécanique en grandes cultures et productions légumières", le CETIOM bénéficie aujourd'hui d'un soutien financier du CASDAR qui émane du ministère de l'Alimentation, de l'Agriculture et de la Pêche. 
Tableau 8. Cas-types de pratiques de désherbage en colza retenus pour la simulation de coûts énergétiques.

\begin{tabular}{|lllll|}
\hline N cas-type & Travail du sol en interculture & $\begin{array}{l}\text { Herbicides } \\
\text { En interculture }\end{array}$ & En culture & Lutte mécanique \\
\hline 1 & 2 déchaumages (profond + superficiel) & Glyphosate $2160 \mathrm{~g} / \mathrm{ha}$ & Devrinol 1.3//Colzor trio 3.5 en plein & - \\
2 & Semis combiné avec outil non animé & Glyphosate $2160 \mathrm{~g} / \mathrm{ha}$ & Devrinol 1.3//Colzor trio 3.5 localisé & 1 binage \\
3 & & Glyphosate $2160 \mathrm{~g} / \mathrm{ha}$ & Colzor trio 3.5 localisé & 1 binage \\
\hline 4 & 1 déchaumage profond/ labour/ & - & Colzor trio 3.5 en plein & - \\
5 & Semis combiné avec outil animé & - & Colzor trio 3.5 localisé & 1 binage \\
6 & & - & - & 2 hersages + 1 binage \\
\hline
\end{tabular}

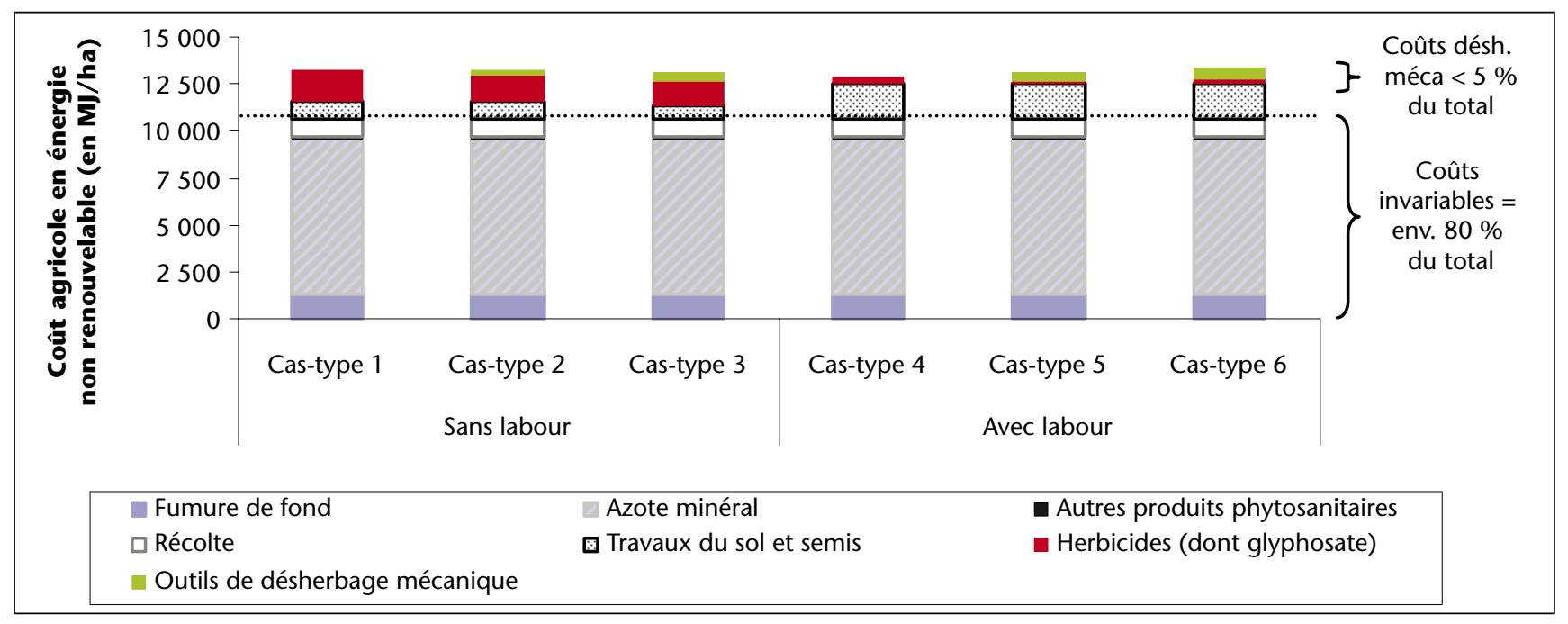

Figure 6. Coûts énergétiques comparés de différentes modalités de désherbage du colza

Hypothèse pour le calcul : sol intermédiaire, apport de $160 \mathrm{U}$ d'azote et 70 de soufre, rendement 35 g/ha, pas d'apport organique, glyphosate $2160 \mathrm{~g} / \mathrm{ha}$ (mod. 1, 2, 3), enfouissement de l'herbicide de présemis par un déchaumage superficiel pour les modalités 1 et 2.

\section{RÉFÉRENCES}

1. Debaeke $P$. Le désherbage intégré en grande culture : bases de raisonnement et perspectives d'application. Cah Agr 1997 ; 6 : 185-94.

2. Munier-Jolain N, Chauvel B, Gasquez J. Stratégies de protection intégrée contre les adventices des cultures : le retour de l'agronomie. Enjeux phytosanitaires pour l'agriculture et l'environnement. Paris : Editions Lavoisier, 2005.

3. Melander B, Rasmussen IA, Bàrberi P. Integrating physical and cultural methods of weed control, examples from European research. Weed Science $2005 ; 53: 369-81$.

4. Duroueix F. Gérer la disparition de la trifluraline. Perspectives Agricoles $2008 ; 347: 60-2$.

5. Duroueix F. Désherbage Colza: la nouvelle donne. Perspectives Agricoles 2009 ; 357 : 85-9.

6. Quéré L. Le désherbage mécanique comme alternative ou complément au chimique. Perspectives Agricoles 2006 ; $325: 60-4$.

7. Quéré L, Lucas JL. Oilseed rape mechanical weed control as an alternative or a complement to chemical weed control. In: International Rapeseed Congress. Wuhan (Chine), 2007.
8. Valantin-Morison M, Guichard L, Jeuffroy MH. Comment maîtriser la flore adventice des grandes cultures à travers les éléments de l'itinéraire technique? Innovations Agronomiques 2008; 3 : 27-41.

9. Melander B. Lutte physique contre les adventices. Phytoma 2006 ; 591 ; 26-9.

10. Bond W, Grundy AC. Non-chemical weed management in organic farming systems. Weed Research $2001 ; 41$ : 383-405.

11. Lucas JL, Estragnat $A$, Jung $L$. Des outils performants. Oleoscope $2004 ; 77$ : 15-6.

12. Lecomte $V$. Le désherbage mixte du tournesol: une technique prometteuse. Oleoscope 2004 ; $77: 10-3$.

13. Pilorgé $E$, Quéré L, Lecomte V. Intérêt des techniques combinées pour le désherbage du tournesol. In: XIXe conférence du COLUMA. Journées internationales sur la lutte contre les mauvaises herbes. Dijon, 2004.

14. Chopard P, Choux G, Courbet E., et al. Des alternatives au désherbage chimique en grandes cultures pour une amélioration de la qualité des eaux : I'expérience franc-comtoise. In: XXe conférence du COLUMA. Journées internationales sur la lutte contre les mauvaises herbes. Dijon, 2007.
15. Lieven J, Quéré L, Lucas JL. Oilseed rape weed integrated management : concern of mechanical weed control. In : Congrès Endure 2008: Diversifying Crop protection. La Grande Motte, 2008.

16. Muchembled C, Royer C, Fallet M, Germain JC, Leclère $T$. Désherbage combiné de la betterave. Résultats de deux années d'expérimentation de désherbage mécanique. In: XXe conférence $d u$ COLUMA. Journées internationales sur la lutte contre les mauvaises herbes. Dijon. 2007.

17. Pingeault N. Améliorer la qualité de l'eau: un indicateur pour favoriser une utilisation durable des produits phytosanitaires. Atelier OCDE, Ministère de l'Agriculture et de la Pêche, France, 2007.

18. Munier-Jolain N, Deytieux V, Guillemin JP, Granger $S$, Gaba $S$. Conception et évaluation multicritères de prototypes de systèmes de culture dans le cadre de la Protection Intégrée contre la flore adventice en grandes cultures. Innovations Agronomiques $2008 ; 3: 75-88$. 